\title{
Efeitos das isoflavonas sobre 0 assoalho pélvico e a vascularização peri-uretral de mulheres na pós-menopausa
}

\author{
Effects of isoflavones on the pelvic floor and the periurethral vascularization \\ of postmenopausal women \\ Leni Aparecida Spagna Accorsi ${ }^{1}$, Mauro Abi Haidar ${ }^{2}$, Ricardo Santos Simões ${ }^{3}$, \\ Alfeu Cornélio Accorsi Neto ${ }^{4}$, Rejane Mosquette ${ }^{5}$, José Maria Soares Junior ${ }^{6}$, Edmund Chada Baracat
}

\section{Resumo}

Objetivo: avaliar a força muscular do assoalho pélvico e os vasos periuretrais de mulheres na pós-menopausa, antes e após seis meses de uso contínuo de extrato de soja. Métodos: estudo prospectivo com 30 mulheres na pós-menopausa antes e após o uso de extrato de soja (100 mg/dia) durante seis meses consecutivos. Foram investigadas a perda urinária e a força muscular do assoalho pélvico por perineômetro digital e avaliação funcional. Avaliou-se ainda o número de vasos da região peri-uretral pela dopplervelocimetria. Para comparar os resultados antes e após tratamento, utilizou-se o teste pareado $t$ de Student. Resultados: das 30 mulheres analisadas, 20 referiram alguma forma de perda urinária no inicio do experimento. A melhora deste sintoma ocorreu em $15(75 \%)$ mulheres após o tratamento. A medida da pressão

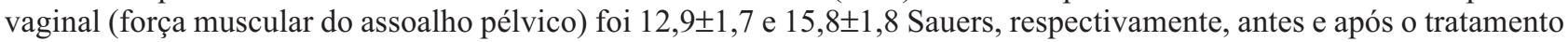
$(\mathrm{p}<0,001)$. Observou-se aumento da pressão em $22(73,3 \%)$ mulheres no final do estudo. Na avaliação funcional, verificou-se que 12 mulheres não sofreram alteração da função muscular do assoalho pélvico, ou seja, 18 (60\%) tiveram aumento da força muscular. Pelo exame ultra-sonográfico (Doppler) obtivemos no início do experimento 2,20 $\pm 0,15$ vasos sanguíneos/campo, passando para 3,4 $\pm 0,2$ vasos sanguíneos/campo ao final do experimento $(\mathrm{p}<0,001)$. Em 21 mulheres $(70 \%)$ registrou-se aumento do número dos vasos peri-uretrais após os seis meses de tratamento. Conclusão: ressalta-se que são resultados preliminares, havendo necessidade de outras investigações com número maior de participantes em estudo duplo-cego, randomizado e controlado por placebo. Contudo, o tratamento com extrato de soja, por seis meses consecutivos, determinaria aumento da força muscular do assoalho pélvico e do número de vasos peri-uretrais em mulheres na pós-menopausa.

PALAVRAS-CHAVE: Isoflavonas/uso terapêutico; Soalho pélvico; Pós-menopausa; Uretra/efeitos de drogras/fisiologia/ ultrasonografia; Fluxometria por laser-doppler

\section{Abstract}

Purpose: to evaluate muscular strength of the pelvic floor and the periurethral vessels of postmenopausal women before and after six months of soybean extract treatment. Methods: the study was conducted on 30 postmenopausal women before and after six consecutive months of soyabean extract (100 mg/day) administration. Urinary loss and muscular strength of the pelvic floor were investigated through digital perineometer and functional evaluation. Digital color Doppler in the periurethral region was used to count the number of vessels. For statistical analysis, the paired Student $t$ test was applied to compare the results before and after the treatment. Results: twenty women reported urinary incontinence before the treatment period. The amelioration of this symptom was observed in $15(75 \%)$ women after the treatment. Vaginal pressure (muscular strength of the pelvic floor) was $12.95 \pm 1.73$ and $15.86 \pm 1.86$ Sauers, before and after the treatment, respectively $(\mathrm{p}<0.001)$. Twenty-two women $(73.3 \%)$ presented an increase in the pressure at the end of this study. In relation to the function evaluation, $18(60 \%)$ had improvement in muscular strength and

1 Pós-graduanda do Departamento de Ginecologia - Universidade Federal de São Paulo UNIFESP - São Paulo (SP), Brasil.

2 Professor Adjunto do Departamento de Ginecologia - Universidade Federal de São Paulo UNIFESP - São Paulo (SP), Brasil.

3 Residente em Ginecologia e Obstetrícia - Universidade Federal de São Paulo UNIFESP - São Paulo (SP), Brasil.

4 Professor Adjunto do Departamento de Ginecologia - Faculdade de Medicina de Catanduva FAMECA - Catanduva (SP), Brasil.

5 Fisioterapeuta, Pós-graduanda do Departamento de Ginecologia - Universidade Federal de São Paulo UNIFESP - São Paulo (SP), Brasil.

6 Professor Adjunto do Departamento de Ginecologia - Universidade Federal de São Paulo UNIFESP - São Paulo (SP), Brasil.

7 Professor Titular do Departamento de Ginecologia da Universidade Federal de São Paulo UNIFESP - São Paulo (SP), Brasil; Professor Titular da Disciplina de Ginecologia da Universidade de São Paulo - USP - São Paulo (SP), Brasil.

Trabalho realizado no Setor de Climatério da Disciplina da Ginecologia da Faculdade de Medicina de Catanduva -FAMECA - Catanduva (SP), Brasil. Correspondência: José Maria Soares Júnior

Rua Sena Madureira 1245 apto. 11 - 04151-021- São Paulo-SP - Tel: (011) 5081-3685 - Fax: (011) 5572-4268 - e-mail: jsoares415@hotmail.com 
12 women did not present any change. On ultrasonography (Doppler), the number of vessels was $2.20 \pm 0.15$ blood vessels/field in the beginning of this study and 3.46 \pm 0.25 blood vessels/field at the end of the treatment $(p<0.001)$. An increase in the number of periurethral vessels was detected in 21 women (70\%).Conclusion: it is important to emphasize that these are preliminary results. A double blind randomized and placebo-controlled clinical trial with a high number of participants is necessary. However, the treatment with concentrated soybean extract (100 mg per day) for six consecutive months may determine an improvement in pelvic floor muscular strength and an increase in the number of periurethral vessels in postmenopausal women.

KEYWORDS: Isoflavone/therapeutic use; Pelvic floor; Postmenopause; Urethra/drug effects/physiology/ultrasonography; Laser-doppler flowmetry

\section{Introdução}

$\mathrm{Na}$ pós-menopausa, a deficiência de estrogênios propicia sintomas desagradáveis que implicam na piora da qualidade de vida. A longo prazo, as manifestações tardias do hipoestrogenismo determinam alterações da pele e dos órgãos urogenitais, bem como o aparecimento de doenças cardiovasculares e osteoporose ${ }^{1}$.

Em relação às alterações dos órgãos urogenitais, além do efeito do hipoestrogenismo, há processos de envelhecimento na região pélvica que determinam enfraquecimento do assoalho pélvico, que levam a diminuição da força e da massa muscular ${ }^{2,3}$. A força muscular depende do tipo de fibra muscular, da vascularização e do trofismo do tecido ${ }^{3,4}$. Assim, a menor vascularização pélvica determina menor irrigação tecidual, contribuindo para a atrofia muscular, ocorrendo diminuição da pressão uretral por perda do coxim vascular sobre a uretra, diminuição da força muscular, havendo a incontinência urinária de esforço ${ }^{4,5}$.

A avaliação do assoalho pélvico é fundamental para identificar problemas no sistema urogenital que possam afetar a qualidade de vida da mulher. Ortiz et al. ${ }^{6}$ estabeleceram uma escala para avaliação da força da musculatura do assoalho da pelve com o uso de perineômetro. Com este método, Moreira ${ }^{7}$ relatou que mulheres com incontinência urinária apresentavam redução significante da função muscular do assoalho pélvico em comparação com mulheres continentes.

Apesar dos benefícios já conhecidos com a terapia hormonal, a adesão é pequena, cerca de 10 a $20 \%^{8}$. Várias alternativas à terapia estroprogestativa estão sendo avaliadas, entre elas as isoflavonas, que apresentam afinidade pelos receptores de estrogênio, que seriam responsáveis pelos seus efeitos agonistas ${ }^{9}$. O interesse no estudo das isoflavonas como alternativa terapêutica advém de vários estudos publicados realçando os efeitos das isoflavonas nos sintomas menopáusicos ${ }^{9,10}$, vagina e endométrio ${ }^{10,11}$ e na mama ${ }^{10}$.
Há vários trabalhos na literatura mostrando o benefício da terapia hormonal clássica sobre a força muscular do assoalho pélvico ${ }^{12}$. Deve-se salientar que a reposição estrogênica em mulheres na pós-menopausa leva a nítido aumento no número de vasos peri-uretrais, os quais foram submetidos à dopplervelocimetria ${ }^{13,14}$. Contudo, não há dados na literatura sobre os efeitos das isoflavonas sobre o assoalho pélvico e a vascularização periuretral. Assim, este estudo piloto tem como objetivo analisar a perda urinária, a força muscular do assoalho pélvico e o número de vasos periuretrais em mulheres na pós-menopausa antes e após seis meses de tratamento com extrato concentrado de soja.

\section{Métodos}

Foram incluídas 30 mulheres na pós-menopausa, atendidas no ambulatório do Setor de Climatério da Disciplina da Ginecologia da Faculdade de Medicina de Catanduva (FAMECA), no período de novembro de 2003 a maio de 2004 . O projeto foi analisado e aprovado pelo Comitê de Ética em Pesquisa da FAMECA. Foram submetidas aos exames do protocolo de pesquisa. esclarecimentos pormenorizados sobre o estudo, concordarem com todos os procedimentos previstos e assinarem o Termo de Consentimento Informado, as pacientes submeteram-se a anamnese e aos exames físico geral e ginecológico minuciosos na fase de pré-seleção. Além disto, as pacientes receberam informações básicas sobre noções anatômicas da função do assoalho da pelve e do equilíbrio vésico-esfincteriano.

Foram incluídas para participar do estudo apenas as que preencherem os seguintes critérios: idade entre 45-55 anos; amenorréia por pelo menos 12 meses, com concentração de hormônio foliculo-estimulante (FSH) superior a $40 \mathrm{mU} / \mathrm{mL}$; não estar fazendo uso de qualquer medicamento hormonal especifico para a sindrome do climatério nos últimos 12 meses; não estar usando soja e seus derivados. Todas deviam apresentar exame 
físico normal e indice de massa corporal (IMC) até $30 \mathrm{~kg} / \mathrm{m}^{2}$ (Tabela 1 ).

Tabela 1 - Características demográficas e clínicas das mulheres participantes do estudo (média e erro padrão da média).

\begin{tabular}{lcc}
\hline Mulheres participantes & Média & EPM \\
\hline Idade (anos) & 53,8 & 0,98 \\
Menarca (anos) & 13,1 & 0,29 \\
Número de filhos & 3,1 & 0,24 \\
Início da menopausa (anos) & 47,8 & 0,73 \\
Tempo de menopausa (anos) & 6,1 & 0,63 \\
Peso (kg) & 63,4 & 1,80 \\
Altura (m) & 1,57 & 1,23 \\
IMC (kg/m) & 25,9 & 1,60 \\
\hline
\end{tabular}

IMC = índice de massa corpórea; EPM = erro padrão da média.

Foram excluídas do estudo as pacientes que apresentavam: neoplasia conhecida ou suspeita de ser estrogênio - dependente; evidência de alterações malignas na mamografia pré-estudo; esfregaço citológico cervical diagnosticado como classe III ou mais; doença renal, tiroidiana ou hepática crônica; acidente vascular cerebral e infarto do miocárdio; distúrbios tromboembólicos (trombose, tromboflebite); diabetes; hipertensão arterial sistêmica não controlada ( $>160 \mathrm{mmHg}$ para sistólica ou $>100 \mathrm{mmHg}$ para a diastólica); e aquelas que não conseguiam contrair o assoalho pélvico.

Todas as medicações e procedimentos concomitantes foram levados ao conhecimento do investigador médico. Já o exame dopplervelocimétrico dos vasos periuretrais foi feito pelo ultra-sonografista, que não tinha acesso às informações sobre a medicação ou outros conhecimentos que pudessem interferir no estudo (cegamento do avaliador). Após a fase de seleção, cada paciente recebeu cápsulas contendo extrato concentrado de soja com $70 \%$ de proteina de soja e $30 \%$ de isoflavonas $100 \mathrm{mg}$ com 66,32 e $2 \%$, respectivamente das isoformas de genisteina, daidzeína e gliceteína -NUTRAMED, São Paulo/SP), sendo que cada paciente recebia 30 cápsulas, devendo tomar uma ao dia por via oral. Além disso, as pacientes anotavam o número de comprimidos ingeridos, bem como eventos adversos que pudessem ter e medicamentos concomitantes no cartão diário. Após 30 dias essas pacientes retornavam para exames periódicos e recebiam mais 30 cápsulas e o cartão era analisado, sendo este procedimento realizado por seis meses. Todas as pacientes, no início e no final do estudo, foram submetidas a exame físico geral e ginecológico. Dentre os dados do exame físico geral, avaliaramse os níveis pressóricos, bem como o peso e altura para determinação do IMC; avaliação subjetiva da perda de urina; avaliação objetiva do assoalho pélvico por perineômetro; avaliação funcional do assoalho pélvico, por palpação digital vaginal (AFA) e avaliação do número de vasos periuretrais por dopplervelocimetria.

Avaliação subjetiva da perda de urina: a perda de urina foi avaliada por meio do relato da paciente quanto à perda de urina antes e após os seis meses da medicação. A avaliação objetiva do grau de função da musculatura do assoalho pélvico foi determinada pela medida da pressão vaginal com o auxílio de perineômetro digital KG 40, da marca Kroman, com graduação de 0 a 100 Sauers. Para a obtenção das medidas, utilizou-se sensor vaginal, revestido com preservativo lubrificado introduzido na vagina da paciente, deixando-se aproximadamente $1 \mathrm{~cm}$ no meio exterior. Esta localização permitiu que a porção localizada a aproximadamente $3,5 \mathrm{~cm}$ do intróito vaginal estivesse em contato com o sensor. No inicio, o aparelho era calibrado e a medida era tomada após a paciente realizar uma contração do assoalho pélvico, visualizando-se no visor a graduação da avaliação da pressão de contração. O uso deste aparelho exige supervisão técnica rigorosa, para garantir que a pressão intra-abdominal não esteja sendo medida, ao invés da contração real do assoalho da pelve. Portanto, as avaliações antes e após os seis meses de tratamento foram realizadas pelo mesmo investigador.

A avaliação funcional consistiu em analisar a função da musculatura do assoalho pélvico, pela visualização da contração desta musculatura e da percepção táctil à palpação digital vaginal (AFA) durante esta contração. A paciente, em posição ginecológica, foi orientada a contrair os músculos perineais e depois, repetia a contração muscular com os dedos indicador e médio do examinador introduzidos na vagina. Baseados neste procedimento as pacientes foram avaliadas da seguinte maneira: Grau 0 - sem contração perineal visível, nem à palpação (ausência de contração); Grau 1 - sem contração perineal visível, contração reconhecível somente à palpação; Grau 2 - contração perineal fraca, contração fraca à palpação; Grau 3 - contração perineal presente e resistência não opositora à palpação; Grau 4 - contração perineal presente e resistência opositora não mantida mais do que cinco segundos à palpação, e Grau 5 - contração perineal presente e resistência opositora mantida mais do que cinco segundos à palpação ${ }^{15}$.

A dopplervelocimetria para avaliação dos vasos periuretrais foi realizada em equipamento ATL- 9/HDI 2000, com transdutor transvaginal setorial de freqüência variável entre 5 e $9 \mathrm{MHz}$. A posição adotada para o exame foi o decúbito dorsal horizontal, em posição subclitoridiana, sem adentrar a vagina. As pacientes encontravam-se com a 
bexiga cheia, o que permitia sua fácil identificação, assim como do colo vesical, da uretra e da borda inferior da sinfise púbica. Estabeleceu-se, como região de estudo, uma área em forma de trapézio, sempre do mesmo tamanho, englobando o colo vesical, a uretra proximal e média, toda a região periuretral e a sinfise púbica. Foram contados os vasos sanguíneos dessa região, caracterizados por pontos brancos sob um fundo azul. Optou-se pelo menor cursor possivel para diminuir a probabilidade de sobreposição de achados de dois vasos próximos.

\section{Análise estatística}

O critério usado para a determinação do tamanho amostral ( $\mathrm{n}=$ número de participantes) foi baseado na lei dos grandes números e no teorema central do limite, que mostra o comportamento da média amostral como muito próximo da distribuição de Gauss para "n" ser considerado grande. Neste estudo, com distribuição das variáveis homogêneas o número de 30 pacientes foi considerado adequado.

Para análise dos dados obtidos foi utilizada análise estatística inferencial, teste $t$ de Student pareado e o coeficiente de correlação de Pearson para as variáveis analisadas antes e após o tratamento $(\mathrm{p} \leq 0,05)$.

\section{Resultados}

$\mathrm{Na}$ avaliação subjetiva das 30 participantes, detectou-se que 20 mulheres $(66,7 \%)$ referiram alguma forma de perda de urina antes do tratamento. Após seis meses de tratamento, 5 pacientes $(16,7 \%)$ referiram perda de urina $(\mathrm{p}<0,001)$.
A avaliação objetiva da pressão vaginal (força muscular do assoalho pélvico) pelo perineômetro registrada no início foi de 12,9 $\pm 1,7$ Sauers e após a terapia com extrato de soja foi $15,8 \pm 1,8$ Sauers $(p \leq 0,001)$. Este aumento ocorreu em 22 mulheres $(73,3 \%)$, sendo que em $5(16,6 \%)$ ocorreu redução da força e $3(10 \%)$ não mostraram alteração após tratamento com extrato de soja (Tabela 2).

$\mathrm{Na}$ avaliação funcional da força muscular do assoalho pélvico, os resultados mostram que houve aumento significante em 18 (60\%) mulheres após o tratamento, entretanto, em 7 (40\%) não se observou nenhuma mudança (Tabela 3).

Em relação à contagem do número de vasos periuretrais pela dopplervelocimetria durante o exame ultra-sonográfico, foi obtido média de $2,2 \pm 0,1$ e 3,4 $\pm 0,2$ vasos/região, respectivamente antes e no final do período de tratamento $(p<0,001)$. Com relação ao número de mulheres, verificou-se que 21 (70\%) mulheres apresentaram aumento no número de vasos, em 3 (10\%) ocorreu diminuição e em 6 (20\%) não ocorreu alteração (Tabela 2).

\section{Discussão}

Representa o climatério o período da vida em que a mulher sofre modificações regressivas decorrentes da redução dos hormônios sexuais, principalmente os estrogênios produzidos pelos ovários ${ }^{16}$. Entre elas destacam-se as alterações do assoalho pélvico ${ }^{13,14}$. O hipoestrogenismo acarreta, em geral, atrofia das estruturas responsáveis pela estática pélvica. Os aparelhos de suspensão e de sustentação, pela insuficiência circulatória, tornam-se frouxos e com menor elasticidade. No-

Tabela 2 - Número e percentagem de pacientes que obtiveram aumento da pressão vaginal (força muscular do assoalho pélvico) e do número de vasos peri uretrais antes e após o uso de extrato de soja durante seis meses.

\begin{tabular}{|c|c|c|c|c|}
\hline & Aumento & Diminuição & Sem alteração & Total \\
\hline Pressão vaginal (Sauers) & $22(73,3 \%)$ & $5(16,6 \%)$ & $3(10 \%)$ & 30 \\
\hline Número de vasos sanguíneos & $21(70,0 \%)$ & $3(10,0 \%)$ & $6(20 \%)$ & 30 \\
\hline
\end{tabular}

Tabela 3 - Distribuição (em graus) da avaliação funcional da função muscular antes e após seis meses de tratamento com extrato de soja.

\begin{tabular}{|c|c|c|c|c|}
\hline \multirow{2}{*}{ Grau } & \multicolumn{4}{|c|}{ Número de pacientes } \\
\hline & Antes do tratamento & Após tratamento & Não sofreram alteração & Sofreram alteração \\
\hline 1 & 9 & 2 & 2 & 7 \\
\hline 2 & 14 & 11 & 6 & 8 \\
\hline 3 & 3 & 8 & 2 & 1 \\
\hline 4 & 3 & 4 & 1 & 2 \\
\hline 5 & 1 & 5 & 1 & 0 \\
\hline Total & 30 & 30 & 12 & 18 \\
\hline
\end{tabular}

Grau 0 - sem contração perineal visível, nem à palpação (ausência de contração); Grau 1 - sem contração perineal visível, contração reconhecível somente a palpação; Grau 2 - contração perineal fraca, contração fraca; Grau 3 - contração perineal presente e resistência não opositora à palpação; Grau 4 - contração perineal presente e resistência opositora não mantida mais do que cinco segundos; Grau 5 - contração perineal presente e resistência opositora mantida mais do que cinco segundos.

Rev Bras Ginecol Obstet. 2006; 28(9): 545-50. 
tam-se, nos diafragmas pélvico e urogenital, redução da massa muscular e aumento do colágeno, justificando o aparecimento de prolapso genital e a incontinência urinária ${ }^{17-19}$.

Os fitoestrogênios têm atividades biológicas semelhantes às dos estrogênios ${ }^{20}$. A ação deles no trato urinário baixo é ainda pouco conhecida. Não existem trabalhos que tenham mostrado possivel relação entre o uso de isoflavonas e alterações nesta região.

Ao efetuarmos a avaliação objetiva da musculatura do assoalho pélvico, pelo perineômetro digital antes e após o uso de $100 \mathrm{mg} /$ dia de extrato de soja durante seis meses, verificamos que houve aumento da força muscular em 73,3\% das mulheres, sugerindo efeito da isoflavona similar aos estrogênios $^{21}$, o que sugere susceptibilidade urogenital a esses hormônios, reforçada pelo achado de receptores hormonais na musculatura pélvica e no trato urinário baixo ${ }^{12}$.

A avaliação funcional do assoalho pélvico foi realizada por ser fundamental pela alta sensibilidade e especificidade deste método, permitindo análise funcional desta musculatura ${ }^{6}$. Em nossa casuística, verificamos que 18 (60\%) mulheres apresentaram melhora significativa após os seis meses de tratamento.

No que tange à avaliação subjetiva sobre a perda de urina, os nossos dados revelaram melhora em 15 (75\%) mulheres. Deve-se associar esta melhora com a maior intensidade de contração deste grupo muscular ${ }^{22}$. Na dopplervelocimetria dos vasos periuretrais, observa-se aumento significante destes vasos, concomitantemente com a melhora

\section{Referências}

1. Masse PG, Tranchant CC, Dosy J, Donovan SM. Coexistence of osteoporosis and cardiovascular disease risk factors in apparently healthy, untreated postmenopausal women. Int J Vitam Nutr Res. 2005;75(2):97-106.

2. Iosif CS, Bekassy Z. Prevalence of genito-urinary symptoms in the late menopause. Acta Obstet Gynecol Scand. 1984;63(3):257-60.

3. Sirola J, Rikkonen T. Muscle performance after the menopause. J Br Menopause Soc. 2005;11(2):45-50.

4. Thompson LV. Effects of age and training on skeletal muscle physiology and performance. Phys Ther. 1994;74(1):71-81.

5. Chmel R, Novackova M, Vlk R, Horcicka L. [Epidemiological aspects of the female urinary incontinence]. Cas Lek Cesk. 2005;144(2):95-7. Review. Czech.

6. Contreras Ortiz O, Coya Nuñez F, Ibañez G. [Evaluación funcional del piso pelviano femenino (classificación dos sintomas urinários. Estudos com terapia estrogênica mostram modificação nos achados da doplervelocimetria dos vasos periuretrais associada com redução de queixas urinárias em mulheres na pós-menopausa ${ }^{19}$. Tais dados sugerem que a isoflavona poderia agir nos vasos periuretrais, de forma semelhante à que foi atribuída por outros autores aos estrogênios ${ }^{9,19,23}$. Uma explicação para este fato seria aumento dos fatores de crescimento vásculoendotelial que estaria relacionado com o receptor de estrogênio. Contudo, estudos experimentais mostram que as isoflavonas atuariam inibindo a expressão deste fator in vitro ${ }^{24}$. É possivel que o mecanismo de aumento dos vasos da pele esteja relacionado com outros fatores celulares que possam envolver o óxido nítrico e a calmodulina ${ }^{25,26}$. Alguns autores atribuem este efeito ao metabólito da daidzeína, o desidroequol ${ }^{25}$. Possivelmente, o aumento dos vasos periuretrais estaria relacionado com melhor nutrição tissular, e possivelmente, com aumento da força muscular do assoalho pélvico e melhora da sintomatologia urinária.

Nosso estudo evidenciou que a terapia com extrato concentrado de soja aumentou a força muscular do assoalho pélvico e o número de vasos da região periuretral, diminuindo episódios de perda urinária em mulheres na pós-menopausa. Contudo, ressalta-se que este experimento é piloto, tendo algumas limitações devido ao desenho do estudo. Apesar de o número de pacientes ser adequado, há necessidade ainda de outras investigações clínicas com estudo duplo cego, randomizado e controlado por placebo, incluindo número maior de mulheres.

funcional)]. Bol Soc Latinoam Uroginecol Cir Vaginal. 1994;1(1):5-9. Spanish.

7. Moreira ECH. Valor da avaliação propedêutica objetiva e subjetiva no diagnóstico da incontinência urinária feminina: correlação com a força muscular do assoalho pélvico. Rev Bras Ginecol Obstet. 2000;22(9):597.

8. Branca F, Lorenzetti S. Health effects of phytoestrogens. Forum Nutr. 2005;(57):100-11.

9. Han KK, Soares JM Jr, Haidar MA, de Lima GR, Baracat EC. Benefits of soy isoflavone therapeutic regimen on menopausal symptoms. Obstet Gynecol. 2002;99(3):389-94.

10.Kaari C, Haidar MA, Junior JM, Nunes MG, Quadros LG, Kemp C, et al. Randomized clinical trial comparing conjugated equine estrogens and isoflavones in postmenopausal women: a pilot study. Maturitas. 2006;53(1):49-58. 
11.Lamartiniere CA. Protection against breast cancer with genistein: a component of soy. Am J Clin Nutr. 2000;71(6 Suppl):1705S-7S.

12.Zhu L, Lang J, Feng R, Chen J, Wong F. Estrogen receptor in pelvic floor tissues in patients with stress urinary incontinence. Int Urogynecol J Pelvic Floor Dysfunct. 2004;15(5):340-3.

13.Girão MJ, Jarmy-Di Bella ZI, Sartori MG, Baracat EC, Lima GR. Doppler velocimetry parameters of periurethral vessels in postmenopausal incontinent women receiving estrogen replacement. Int Urogynecol J Pelvic Floor Dysfunct. 2001;12(4):241-6.

14.Long CY, Liu CM, Hsu SC, Chen YH, Wu CH, Tsai EM. A randomized comparative study of the effects of oral and topical estrogen therapy on the lower urinary tract of hysterectomized postmenopausal women. Fertil Steril. 2006;85(1):155-60.

15. Contreras Ortiz O, Coya Nuñez F. Dynamic assessment of pelvic floor functionin women using the intravaginal device test. Int Urogynecol $\mathrm{J}$ Pelvic FloorDysfunct. 1996;7(6):317-20.

16. Silva Filho CR, Baracat EC, Conterno LO, Haidar MA, Ferraz MB. Climacteric symptoms and quality of life: validity of women's health questionnaire. Rev Saúde Publica. 2005;39(3):333-9.

17.Girão MBC, Sartori MGF, Baracat EC, Lima GR. Cirurgia vaginal e uroginecologia. 2a ed. Porto Alegre: Artes Médicas; 2002. p. 105-34.

18.Sartori MG, Girão MJ, de Jesus Simões M, Sartori JP, Baracat EC, Rodrigues de Lima G. Quantitative evaluation of collagen and muscle fibers in the lower urinary tract of castrated and under-hormone replacement female rats. Clin Exp Obstet Gynecol. 2001;28(2):92-6.
19. Cardozo L, Lose G, McClish D, Versi E. A systematic review of the effects of estrogens for symptoms suggestive of overactive bladder. Acta Obstet Gynecol Scand. 2004;83(10):892-7.

20.Slomczynska M. The effect of phytoestrogens on the reproductive tract. Pol J Vet Sci. 2004;7(3):223-6.

21. Glazer HI, Romanzi L, Polaneczky M. Pelvic floor muscle surface electromyography. Reliability and clinical predictive validity. J Reprod Med. 1999;44(9):779-82.

22.Klarskov P, Belving D, Bischoff N, Dorph S, Gerstenberg T, Okholm B, et al. Pelvic floor exercise versus surgery on the female urinary stress incontinence. Urol Int. 1986;41(2):129-32.

23.Battaglia C, Mancini F, Persico N, Penacchioni P, Regnani G, Volpe A, et al. Tibolone, oral or transdermal hormone replacement and colour Doppler analysis: a prospective, randomised pilot study. Maturitas. 2004;48(4):446-55.

24.Trompezinski S, Denis A, Schmitt D, Viac J. Comparative effects of polyphenols from green tea (EGCG) and soybean (genistein) on VEGF and IL-8 release from normal human keratinocytes stimulated with the proinflammatory cytokine TNFalpha. Arch Dermatol Res. 2003;295(3):112-6.

25.Chin-Dusting JP, Boak L, Husband A, Nestel PJ. The isoflavone metabolite dehydroequol produces vasodilatation in human resistance arteries via a nitric oxide-dependent mechanism. Atherosclerosis. $2004 ; 176(1): 45-8$.

26.Woodman OL, Missen MA, Boujaoude M. Daidzein and 17 beta-estradiol enhance nitric oxide synthase activity associated with an increase in calmodulin and a decrease in caveolin-1. J Cardiovasc Pharmacol. 2004;44(2):155-63. 